\title{
A generalization of a theorem of Aquaro
}

\section{C.E. Aull}

In this paper we introduce the concept of a $\delta \theta$-cover to generalize Aquaro's Theorem that every point countable open cover of a topological space such that every discrete closed family of sets is countable has a countable subcover. A $\delta \theta$-cover of a space $X$ is defined to be a family of open sets $V=U_{n} V_{n}$ where each $V_{n}$ covers $X$ and for $x \in X$ there exists $n$ such that $v_{n}$ is of countable order at $x$. We replace point countable open cover by a $\delta \theta$-cover in Aquaro's Theorem and also generalize the result of Worrell and Wicke that a $\theta$-refinable countably compact space is compact and Jones' result that an $\aleph_{1}$-compact Moore space is Lindelöf which was used to prove his classic result that a normal separable Moore space is metrizable, using the continum hypothesis.

In this paper we introduce the concept of a $\delta \theta$-cover to generalize Aquaro's Theorem [1] that every point countable open cover of a topological space such that every discrete closed family of sets is countable has a countable subcover. We replace point countable open cover by a $\delta \theta$-cover and also generalize the result of Worrell and Wicke [8] that a $\theta$-refinable countably compact space is compact and Jones' [6] result that an $\aleph_{1}$-compact Moore space is Lindelöf which was used to prove his classic result that a normal separable Moore space is metrizable, using the continuum hypothesis.

Received 16 March 1973. 
DEFINITION 1. A $\delta \theta$-cover of a space $X$ is a family of open sets $V=V_{n} V_{n}$ where each $V_{n}$ covers $X$ and for $x \in X$ there exists $n$ such that $V_{n}$ is of countable order at $x$. A space $X$ is said to be $\delta \theta$-refinable if every open cover is refined by a $\delta \theta$-cover.

THEOREM 1. Let every closed discrete family of sets of a space $X$ be countable. Then every $\delta \theta$-cover has a countable subcover.

We use a series of lemmas to establish the theorem.

DEFINITION 2. A set $M$ is distinguished with respect to an open cover $u$ of a topological space $X$ if for $x, y \in M, x \neq y$, $x \in U \in U \Rightarrow y \notin U$.

From Definition 2, Lemma 1 follows.

LEMMA 1. A distinguished set is discrete.

DEFINITION 3. A set $M$ is maximally distinguished with respect to an open cover of a topological space $X$ on a set $H$ if $M \subset H, M$ is distinguisined and if $P$ is distinguished and $M \subset P \subset H$ then $P=M$.

LEMMA 2. If a distinguished set $M$ with respect to an open cover $u$ is contained in a set $H$ then it is contained in a maximally distinguished set on $H$ with respect to $U$.

Proof. The union of any chain of distinguished sets on $H$ with respect to $U$ is a distinguished set on $H$. Hence by the maximal principle there exists a maximal distinguished set on $H$ with respect to $U$ containing $M$.

LEMMA 3. Let $w$ consist of the subfarily of an open cover $u$ that intersects a maximally distinguished set $M$ on $H$ with respect to $U$. Then $\omega$ covers $H$.

Proof. Suppose there exists. $z \in H$, such that there does not exist $W \in W$ such that $z \in W$. Then $M U[z]$ is a distinguished set with respect to $U$ on $H$, and $M \cup[z]$ properly contains $M$ contrary to $M$ being maximal.

Moore [7] obtained a discrete set on the space with the property of the distinguished set in Lerme 3 in a different manner. 
Proof of Theorem 1. Let $H_{n}$ consist of the points of countable order with respect to $v_{n}$ of Definition 1 . Let $w_{n}$ consist of the members of $V_{n}$ that intersect $M_{n}$, a maximal distinguished set on $H_{n}$ with respect to $V_{n}$. By the condition of the theorem and Lemma $1, M_{n}$ is countable, so that $w_{n}$ is also countable. By Lemma $3, w=U w_{n}$ is a countable cover of $X$.

COROLLARY 1. If a $T_{1}$ space is $N_{1}$-compact and satisfies any of the following properties, it is Lindelöf:

(a) so-refinable;

(b) 0-refinable;

(c) meta-Lindelöf (Aquaro [1]);

(d) metacompact or paracompact (Arens and Dugundji [2]);

(e) subparacompact (Christian [5]);

(f) developable (Jones [6]).

Proof. In a $T_{1}$-space $\aleph_{1}$-compactness is equivalent to every closed discrete set being countable. Then $(a),(b),(c)$ and $(d)$ are immediate and (e) follows from Burke's [4] result that a subparacompact space is $\theta$-refinable and $(f)$ follows from the result of Worrell and Wicke [8] that Moore spaces are $\theta$-refinable.

COROLLARY 2. A countable compact space satisfying any of the conditions (a) through ( $f$ ) is compact.

Corollary 2 (b) is due to Worrell and Wicke [8], and Boyte [3] has given a short proof of Corollary 2 (c).

COROLLARY 3. An $\aleph_{1}$-compact $T_{3}$ space is metrizable iff it has a o-locally countable base. 


\section{References}

[1] G. Aquaro, "Point countable open coverings in countably compact spaces", General topology and its relations to modern analysis and algebra. II., 39-41 (Proc. 2nd Prague Topological Symposium, 1966; Academia [Publishing House of the Czechoslovak Academy of Sciences ], Prague, 1967).

[2] Richard Arens and J. Dugundjl, "Remark on the concept of compactness", Portugal. Math. 9 (1950), 141-143.

[3] J.M. Boyte, "A note on compactness and metacompactness", Proc. Japan Acad. 46 (1970), 1084-1085.

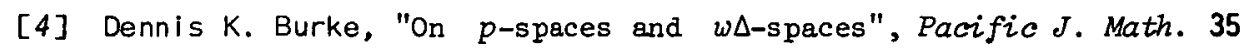
(1970), 285-296.

[5] U.J. Christian, "Concerning certain minimal cover refinable spaces", Fund. Math. 76 (1972), 213-222.

[6] F.B. Jones, "Concerning normal and completely normal spaces", BuZZ. Amer. Math. Soc. 43 (1937), 671-677.

[7] R.L. Moore, Foundations of point set theory (Colloquium Publ. 13. Amer. Math. Soc., Providence, Rhode Island, 1962).

[8] J.M. Worrell, Jr and H.H. Wicke, "Characterizations of developable topological spaces", Canad. J. Math. 17 (1965), 820-830.

Department of Mathematics,

Virginia Polytechnic Institute and State University,

Blacksburg,

Virginia,

USA. 